\title{
CHRONIC UNPREDICTABLE STRESS-INDUCED BEHAVIORAL AND ELECTROPHYSIOLOGICAL ALTERATIONS AND ITS AMELIORATIVE EFFECT BY MYRICETIN MICROEMULSION
}

\author{
SAKTHIVEL G, DEVA KARUNYA M, PRAJISHA P, KEERTHIPRIYA CS AND RAVINDRAN R*
}

Department of Physiology, Dr. A.L.M Post Graduate Institute of Basic Medical Sciences, University of Madras, Chennai - 600 113, Tamil Nadu, India. Email: ravindran.unom@gmail.com

Received: 27 October 2017, Revised and Accepted: 29 November 2017

\section{ABSTRACT}

Objective: The present study is designed to investigate the effects of chronic unpredictable stress (CUS) on electrophysiological and behavioral alterations in male Wistar albino rats and its ameliorating effect by myricetin-microemulsion (MYR-ME).

Materials and Methods: Adult Wistar male albino rats were exposed to CUS for 21 days and treated with MYR-ME (10 mg/kg) for 21 days by oral administration. All the experimental animals were tested for anxiety and cognitive behavior by open-field behavior, light/dark test, eight-arm radial maze, spontaneous alteration T-maze, novel object recognition test, plasma corticosterone level, and electrophysiological activity.

Results: The rats which were exposed to CUS showed memory impairment, increased anxiety, decreased novel explorations, deleterious effect on decision-making, increased corticosterone level, increased brain wave frequency and amplitude, and also heart rate. Whereas, CUS with MYRME-treated group showed a protective effect against CUS-induced behavioral alterations, electrophysiological activity, and corticosterone levels, which is characterized by the enhancement of cognitive function, decreased anxiety and improved decision-making, novel exploration, decreased corticosterone, and electrophysiological activity.

Conclusion: From the present study, it is shown that MYR-ME may act as a potential anxiolytic and nootropic compound against CUS-induced alterations.

Keywords: Chronic unpredictable stress, Myricetin-microemulsion, Cognitive behavior, anxiety, Learning and memory, Electrophysiology.

(c) 2018 The Authors. Published by Innovare Academic Sciences Pvt Ltd. This is an open access article under the CC BY license (http://creativecommons. org/licenses/by/4. 0/) DOI: http://dx.doi.org/10.22159/ajpcr.2018.v11i3.23364

\section{INTRODUCTION}

Stress is a pervasive and unavoidable component in each day of life. Hans Selye defines the term stress as "the non-specific response of the body to any demand for change." Chronic stress exposure of animals is often associated with maladaptation. Depending on the intensity and duration of the stressors, it can affect the cognitive process [1]. The potential negative outcome associated with chronic stress exposure is the development of learning and memory impairment, behavioral changes, mental illnesses, including anxiety, depression, drug addiction, or affective disorders [2]. Normal structure and function of the brain are altered by severe and/or chronic stress. The hippocampus, amygdala, and prefrontal cortex are the areas involved in the neural circuitry mediating stress responses. The hippocampus provides negative feedback regulation of the stress response, particularly to degenerative changes caused by chronic stress [3]. The elevated level of glucocorticoids (GC) was observed in response to stress, which is regulated and maintained by hypothalamic-pituitary-adrenocortical (HPA) axis. GC and excitatory amino acids play the key role in the stress-induced adverse effects. Excess glucocorticoid level increases the intracellular glutamate level which causes the subsequent activation of $\mathrm{N}$-methyl-D-aspartate receptors, which triggers the excess influx $\mathrm{Ca}^{2+}[4]$. In addition, presynaptic release of glutamate by increased GC and subsequent activation of AMPA (Alpha-amino-3-hydroxy-5-methyl-4-isoxazole propionic acid) and NMDA receptors, followed by the activation of a variety of kinases (e.g., CaMKII, PKC, PKA, and ERK/MAPK) and downstream signaling pathways leads to structural modifications at relevant synapses - such as changes in spine shape and length or the formation of new dendritic spines [5], which may be reason to stress-induced deleterious effect.

Many studies have also correlated stress and behavioral alterations. 21 days of chronic unpredictable stress (CUS)-exposed animals showed anxiety, cognitive behavioral changes, and learning and memory impairment in the eight-arm radial maze [6], T-maze [7], and novel object recognition [8], and open-field behavior is used to detect locomotor, anxiety, or increased fear condition in rodents [1]. The light/dark test is based on the innate aversion of rodents to brightly illuminate areas and on the spontaneous exploratory behavior of rodents in responses to mild stressors that is novel environment and light [9]. From the above studies, it has been cleared that behavioral paradigms are the well-defined marker to assess anxiety-like behavior and cognitive dysfunction in the laboratory animals in CUS-induced model.

Stress has tended to affect central nervous system (CNS) as well as peripheral nervous system, including autonomic nervous system (ANS). In recent days, heart rate variability (HRV) has been used as a biomarker of ANS activity associated with mental stress [10], and stressinduced brain electrical activity is measured by electroencephalogram (EEG) [11].

Latest nature report says that there is a lack of new drug development for CNS disorder for the past two decades [12]. In the recent years, polyphenols have received a great attention as a source of antioxidant supplementation for treating many disease conditions. Myricetin (MYR) $(3,3$, 4', 5, 5', 7-hexahydroxyflavone) is present in many plantderived foodstuffs, notably in grapes, berries, onions, walnuts, herbs, wines, vegetables, fruits, and medicinal plants and is classified as a flavonoid with strong antioxidant effects [13], and this may due to consist of more number of replaceable hydrogen atom present in the benzene ring. Previous studies reported that MYR exhibits a variety of health beneficial effects such as hypoglycemic [14], antioxidant [15], anticancer effect, anti-inflammatory, and antiviral activities and 
also from 1 to $5 \mathrm{mg} / \mathrm{kg}$ body weight which exerts many biological actions [16]. Earlier studies reported that MYR with $10 \mathrm{mg} / \mathrm{kg}$ body weight acted as an analgesic [17] and anxiolytic [18] effect.

MYR has received great attention due to its varied biologically diverse activities. However, due to its slightly polar soluble nature it is inaccessible cross the blood-brain barrier (BBB), which may limit the curative effect of drug. Hence, many biologically active compounds are inaccessible to the brain due to these reasons. Hence, it is necessary to create alternative and efficacious formulation system to delivery MYR [19]. To increase the bioavailability of the drug in the brain, the drug is administrated in the form of a microemulsion (ME). The ME concept was introduced by Hoar and Schulman who generated a ME system using oil-alkali metal soap with water, which is the oil in water emulsions [20]. The earlier pharmacokinetic study reported that MYRME showed (16.05 fold) increased bioavailability when compared to MYR alone after oral administration [21]. Hence, it would be better alternative drug delivery system to target to the brain.

Hence, we hypothesize that MYR in the form of ME formulation may prevent or delay the onset of cognitive dysfunction, behavioral alteration, and electrophysiological activities induced by CUS.

\section{METHODS}

\section{Chemicals and reagents}

MYR and urethane were purchased from Sigma-Aldrich in India, and all other chemicals used are of an analytical grade, purchased from Sisco Research Laboratories, India.

\section{Animals and maintenance}

Adult male Wistar albino rats weighing 200-220 g were used for the present study. They were maintained at controlled room temperature $23 \pm 2{ }^{\circ} \mathrm{C}$ with $12: 12 \mathrm{~h}$ light:dark cycle and the animals were fed with standard rat pellet diet and drinking water ad libitum, except during the CUS procedure. All the experimental protocols were approved by the Institutional Animal Ethical Committee and CPCSEA, University of Madras.

\section{Experimental design}

The animals were randomly divided into four groups, and each group consists of six animals. Group I - Control; Group II - ME formulation administered without MYR; Group III - CUS-exposed animals. and Group IV - CUS+MYR-ME treated.

\section{CUS model}

CUS is an experimental procedure in which animals are exposed to varying unpredictable stressor each day, a slight modification of previously described method [22]. The unpredictable stress model was designed to maximize the unpredictable nature of the stressors and the same in random order. Day 1: Restraint stress: $6 \mathrm{~h}$ the rats were kept immobilized using wire mesh restraint cage, Day 2: Noise-induced stress: $4 \mathrm{~h}$ noise at the level of $100 \mathrm{db}$ were induced uniformly throughout the cage, Day 3: Temperature fluctuation induced stress like cold environment isolation: $15 \mathrm{~min}$ (rats are individually kept in a box containing ice maintained at $4^{\circ} \mathrm{C}$ ), Day 4: Food deprivation (24 h), Day 5: Water deprivation (24 h), Day 6: Overnight lights illumination stress (12 h), and Day 7: Continuous dark (12 h) at day. Group III animals alone were exposed to CUS for 21 days.

\section{MYR ME preparation}

The MYR-space MYR-ME $(\mathrm{O} / \mathrm{W})$ was prepared by dissolving Tween 80 and Tween 20 in 1:2 ratio (surfactant) in ethanol (co-surfactant), then mixed with oleic acid (oil phase), and subsequently, appropriate amount of MYR was added into the mixture. The formulations were formed spontaneously at room temperature when the suitable amount of phosphate buffer ( $\mathrm{pH}$ 6.5) was added by gently vibrating as previously described by Wang et al. MYR-ME was administrated $10 \mathrm{mg} /$ kg orally for 21 days before an hour of CUS exposure. Vehicle groups receive the mixture of Tween 20, Tween 80 (1:2), oleic acid, and ethanol mixed with phosphate buffer ( $\mathrm{pH} 6.5$ ).

\section{Behavioral analysis for anxiety and locomotor activity Open-field test}

Open-field test is used to measure the normal exploratory behavior, locomotor activity, and inherent anxiousness to new open place [23]. The apparatus consists of a rectangular box $(100 \mathrm{~cm} \times 100 \mathrm{~cm}), 40 \mathrm{~cm}$ height fabricated in plywood, and the floor consists of a clean plastic material with a grid painted in black dividing the field into $25(5 \times 5)$ equal squares. The entire task was video recorded for further analysis. The rats were placed in the center of the open-field arena and allowed to freely explore for $5 \mathrm{~min}$. The animals initially spend or explore the peripheral portion of the open field. As it overcomes their initial anxiety, it explores in the central portion of the open field. The time spent in the central squares, peripheral squares rearing, and grooming was recorded and analyzed. A rearing is whenever the rat stood on its hind paws with the elevated body, whereas licking or scratching itself in stationary is grooming. At every consecutive task, the open-field arena is cleaned with $70 \%$ alcohol and dried.

\section{Light/dark test}

The light/dark box is used to assess the anxiety-like behavior of rodents. The box was divided into two compartments, $18 \times 15 \times 15^{\prime \prime}$ (long, wide and high) light compartment with open at the top and $12 \times 15 \times 15^{\prime \prime}$ (long, wide and high) the dark compartment is fully enclosed. The divider between two compartments contains $3 \times 4$ " (wide, high) opening at floor level. This allows the animal entry between the compartments. At the beginning of testing, each animal was placed in the center of the light compartment, and the behavior was videotaped for $5 \mathrm{~min}$ and analyzed. As the rats are nocturnal in nature, they prefer dark compartment to light compartment, thought they are curious to novel environment (light compartment), the shuttling time between the dark/light arenas are recorded such as follows: (1) Initial latency to enter the dark compartment, (2) time spent in the bright area, and (3) time spent in a dark compartment [24]

\section{Behavioral analysis to assess the cognitive function Eight-arm radial maze}

Radial arm maze evaluates the spatial learning and memory in rats, which is one of the important tasks to show the role of hippocampus [25]. The apparatus consists of a central platform (octagonal shape) of $33.5 \mathrm{~cm}$, and eight radiating arms form the central platform of $60 \mathrm{~cm}$ long and $12 \mathrm{~cm}$ wide. The apparatus is kept at $1 \mathrm{~m}$ above from the floor on a table. Spatial (visual) cues are placed outside the apparatus. Maximum time to complete the task is $5 \mathrm{~min}$, and animals were acclimatized to RAM. The acclimatized animals were deprived of food before each test session and trained to take the feed (Reward) kept at the end of alternative arms. In the due course of time, animals with the help of visual cues identify the baited arm. Entry into the arms was recorded for later analysis. The maze was cleaned with $70 \%$ alcohol to prevent odor cues between trails. Time taken to complete the task (i.e., taking feed from the four baited arms) is recorded as latency, entry into unbaited arms counted as reference memory errors, and entry into the baited arm again after eating the feed as working memory error.

\section{Spontaneous alteration T-maze}

Spontaneous alteration T-maze is used to assess cognitive ability and memory retention in rodents. The test is based on the left and right discrimination, and the rats have to discriminate either left or right arm of the T-maze to get the food reward. The T-maze consists of a start box $(12 \mathrm{~cm} \times 12 \mathrm{~cm})$, stem $(35 \mathrm{~cm} \times 12 \mathrm{~cm})$, and two arms $(35 \mathrm{~cm} \times 12 \mathrm{~cm})$, and each arm had a goal area of $(15 \mathrm{~cm} \times 12 \mathrm{~cm})$ and the side walls were of $40 \mathrm{~cm}$ height. The animals were habituated to apparatus and allow to explore in the T-maze for $10 \mathrm{~min}$, during the training session both the arms were baited with sucrose pellets. In the task, a single arm of a T-maze is baited with a small reward (sucrose pellet), the rat is allowed to explore and find the reward. On the following task, the opposing arm 
of the maze is baited. The pattern is repeated for 10 trails. The number of days to attain $80 \%$ correct is often used as a successful performance. Followed by the $80 \%$ corrected response, the rats were tested for memory retention after 2 days [26].

\section{Novel object recognition test}

The test is based on the novelty preference; the test is used to analyze recognition memory. The $1^{\text {st }}$ day animals were habituated to the openfield ( $40 \mathrm{~cm} \times 40 \mathrm{~cm} \times 49 \mathrm{~cm}$ ) apparatus until it freely moves in the open field. On the $2^{\text {nd }}$ day, animals were familiarized with identical objects for 5 min (Trail 1 or T1), where the objects placed diagonally opposite in the central squares of the open field. The arena is cleaned well with $70 \%$ alcohol before any trail. After $2 \mathrm{~h}$ of time interval, one familiar object is replaced with a novel object (trail 2 or T2), and now, the time spent in exploring the objects was recorded. Percentage of time spent was calculated as $\mathrm{T}_{\text {Novel }} /\left(\mathrm{T}_{\text {Novel }}+\mathrm{T}_{\text {Familiar }}\right) \times 100$, where $\mathrm{T}_{\text {Novel }}$ is time spent with a novel object and $\mathrm{T}_{\text {Familiar }}$ is time spent with a familiar object. Successful performance at the task is assessed by relative exploration of the novel object relative to total exploration for both objects [27].

\section{Electrophysiological studies}

\section{Electrocardiography (ECG)}

ECG was recorded after the experimental procedure under the mild urethane (1 g per body $\mathrm{kg}$ ) anesthetized rats using a BioPac MP100 system. The ECG signal was analyzed using Acqknowledge 3.7.3 software (Biopac Systems, BIOPAC Inc., Goleta, CA, USA). The most commonly used approach to measure HRV is time domain analysis, and the data obtained are HR, R-R intervals, and the root mean square of successive differences of adjacent RR intervals (RMSSD) [28].

\section{EEG}

The electrode was implanted in all animals after the end of the experimental procedure under slight anesthetic condition (urethane $1 \mathrm{~g}$ per body $\mathrm{kg}$ ). The animals were fixed on the stereotaxic frame, after aseptic cleaning procedures, a small incision was made in the skin and a recording electrode fixed on the surface of the scalp using dental acrylic resin. The post-operative care was performed according to the ethical guidelines with the administration of saline and to prevent fluid loss during incision and antibiotic to prevent sepsis. After 2 days, the brain electrical activity was recorded using recorder and medicare systems (RMS) EEG-24 brain new-plus (RMS). Signals were filtered between 1 and $70 \mathrm{~Hz}$. Recordings were analyzed by RMS EEG-24 Super sec version 1.1 and expressed in terms of amplitude $(\mu \mathrm{v})$ and frequency $(\mathrm{Hz})$. All experiments were performed at the same time of day (8:00:0012:00 am) to eliminate variation due to circadian rhythms [29].

\section{Plasma corticosterone estimation}

Rat plasma corticosterone level was analyzed using ELISA kit as per manufacture's instruction given in the manual, purchased from CUSABIO, Hubei Province, China.

\section{Statistical analysis}

Data were analyzed using SPSS 20 version software, presented in the form of the bar diagram and tables, and expressed as mean \pm standard error of mean (SEM). One-way ANOVA followed by the multiple comparisons by Tukey's post hoc multiple comparisons was performed. The value $\mathrm{p}<0.05$ was considered as statistically significant. The significance level was represented as "a" when compared to control group, " $b$ " when compared to vehicle group, "c" when compared to CUS group, and "d" when compared to CUS with MYR-ME-treated group.

\section{RESULTS}

\section{Open-field test}

There was a significant difference between the groups in the central square entries as determined by one-way ANOVA $(\mathrm{F}(3,20)=74.34$, $\mathrm{p}=0.021$ ), the results have been summarized in Table 1 . Tukey's post hoc test revealed that central square entry was significantly decreased in CUS-exposed group $(2.72 \pm 0.40, \mathrm{p}=0.001)$ when compared to control $(8.66 \pm 0.55, \mathrm{p}=0.008)$ and vehicle group $(8.33 \pm 0.42, \mathrm{p}=0.005)$. Whereas, CUS + MYR-ME-treated group showed increased $(11.33 \pm 0.66, p=0.018)$ central square entry when compared to control and CUS group.

The peripheral square entries were markedly varies among the groups $(F(3,20)=122.40, p=0.032)$. The increased peripheral square entries were observed in CUS-exposed animals $(63.67 \pm 1.64, p=0.007)$ when compared to control $(33.00 \pm 1.06, \mathrm{p}=0.017)$ and vehicle $(33.33 \pm 1.68$, $\mathrm{p}=0.007)$ groups. Peripheral square entries were decreased in CUS+MYR-ME (22.16 $\pm 1.9, \mathrm{p}=0.012)$-treated group when compared to CUS-exposed group.

The number of grooming was significantly differ among the groups $(F(3,20)=133.98, p=0.002)$. Grooming was increased in the CUS-exposed animals $(50.67 \pm 3.15, p=0.028)$ when compared to control $(8.00 \pm 0.63$, $\mathrm{p}=0.014)$ and vehicle $(7.66 \pm 0.6, \mathrm{p}=0.010)$ groups, this was significantly decreased in CUS+MYR-ME $(16.16 \pm 1.35, \mathrm{p}=0.019)$ treated group.

Rearing showed marked changes between the groups $(F(3,20)=123.20$, $\mathrm{p}=0.009)$. CUS-exposed animals showed significantly increased number of rearing $(46.67 \pm 1.20, p=0.007)$ when compared to control $(19.67 \pm 1.45, \mathrm{p}=0.006)$ and vehicle $(18.67 \pm 1.38, \mathrm{p}=0.011)$ groups. Whereas, CUS+MYR-ME-treated group showed decreased rearing (14.67 $\pm 1.22, \mathrm{p}=0.004)$ when compared to CUS-exposed group.

The number of fecal pellets also showed variation among the groups $(F(3,20)=5.68, p=0.006)$. CUS-exposed animals showed significantly increased number of fecal pellets that emphasize about anxiety (3.67 $\pm 0.41, \mathrm{p}=0.006)$ when compared to control $(1.5 \pm 0.22)$ and vehicle $(1.33 \pm 0.21)$. Whereas, CUS+MYR-ME-treated group showed decreased rearing proving the efficacy of treatment $(1.1 \pm 0.22, p=0.006)$ when compared to CUS-exposed group.

\section{Light/dark test}

There was a statistically significant difference in the initial latency taken to enter in the dark area between groups as determined by oneway ANOVA $(F(3,20)=10.58, p=0.042)$, this is shown in Fig. 1a. Tukey's post hoc test revealed that CUS-exposed animals showed significant decreased $(11.66 \pm 1.70 \mathrm{~s}, \mathrm{p}=0.001)$ time spent in dark compartment, when compared to control $(26.00 \pm 1.77 \mathrm{~s}, \mathrm{p}=0.01)$ and vehicle $(25.33 \pm 2.45 \mathrm{~s}, \mathrm{p}=0.01)$ groups. Time spent in the light compartment was significantly increased in CUS+MYR-ME-treated $(23.83 \pm 2.27 \mathrm{~s}$, $\mathrm{p}=0.003$ ) group when compared to CUS-exposed group.

Table 1: Open-field behavior

\begin{tabular}{lllll}
\hline Parameters & CON & VEH & CUS & CUS+MYR-ME \\
\hline Central squares entries (count) & $8.66 \pm 0.55$ & $8.33 \pm 0.42^{\mathrm{c}, \mathrm{d}}$ & $2.72 \pm 0.40^{\mathrm{a}, \mathrm{b}, \mathrm{d}}$ & $11.33 \pm 0.66^{\mathrm{a}, \mathrm{b}, \mathrm{c}}$ \\
Peripheral squares entries (count) & $33.00 \pm 1.06$ & $33.33 \pm 1.68^{\mathrm{c}, \mathrm{d}}$ & $63.67 \pm 1.64^{\mathrm{a}, \mathrm{b}, \mathrm{d}}$ & $22.16 \pm 1.92^{\mathrm{a}, \mathrm{b}, \mathrm{c}}$ \\
Number of grooming (count) & $8.00 \pm 0.63$ & $7.66 \pm 0.60^{\mathrm{c}, \mathrm{d}}$ & $50.67 \pm 3.15^{\mathrm{a}, \mathrm{b}, \mathrm{d}}$ & $16.16 \pm 1.35^{\mathrm{a}, \mathrm{b}, \mathrm{c}}$ \\
Number of rearing (count) & $19.67 \pm 1.45$ & $18.67 \pm 1.38^{\mathrm{c}, \mathrm{d}}$ & $46.67 \pm 1.20^{\mathrm{a}, \mathrm{b}, \mathrm{d}}$ & $14.67 \pm 1.22^{\mathrm{c}}$ \\
Number of fecal pellets (count) & $1.5 \pm 0.22$ & $1.33 \pm 0.21$ & $3.67 \pm 0.41^{\mathrm{a}, \mathrm{b}, \mathrm{d}}$ & $1.1 \pm 0.22^{\mathrm{c}}$ \\
\hline
\end{tabular}

Describes parameters studied in the open-field behavior. The data were expressed as mean \pm SEM. The data were expressed as mean \pm SEM. "a" represents significance

level when compared to control, "b" represents compared to vehicle, "c" compared to unpredictable stress group, and "d" represents compared to stress with

MYR-ME-treated and the value $\mathrm{p}<0.05$ is considered as statistically significant. SEM: Standard error of mean, CUS: Chronic unpredictable stress, MYR: Myricetin,

ME: Microemulsion 
The time spent in the bright area was different between the groups $(F(3,20)=32.43, p=0.004)$, this is depicted in Fig. 1b. The CUS-exposed group animals showed a marked decreased time spent $(21.66 \pm 1.92 \mathrm{~s}$, $\mathrm{p}=0.008)$ in light area when compared to control $(74.50 \pm 6.26 \mathrm{~s}$, $\mathrm{p}=0.003)$ and vehicle $(75.00 \pm 4.40 \mathrm{~s}, \mathrm{p}=0.002)$ groups, whereas CUS+MYR-ME-treated animals showed significantly increased time spent $(84.00 \pm 6.35 \mathrm{~s}, \mathrm{p}=0.002)$ in light area when compared to CUSexposed group.

Time spent in the dark area showed marked difference in groups $(F(3,20)=31.55, p=0.01)$, which is shown in Fig. 1c. CUS animals showed increased time spent in dark $(272.00 \pm 4.71 \mathrm{~s}, \mathrm{p}=0.007)$ when compared to control $(197.83 \pm 4.83 \mathrm{~s}, \mathrm{p}=0.009)$ and vehicle $(199.66 \pm 4.60 \mathrm{~s}$, $\mathrm{p}=0.006)$, but there was decrease $(191.33 \pm 10.79 \mathrm{~s}, \mathrm{p}=0.003)$ in CUS+MYR-ME-treated group when compared to CUS-exposed group.

\section{Eight-arm radial maze}

One-way ANOVA determined that there is a significant difference in the number of reference memory error (entry into the non-baited armed) among the groups $(F(3,20)=13.43, p=0.01)$, this is shown in Fig. 2 a. The multiple comparison with Tukey's post hoc test revealed that the reference memory error was significantly increased in CUS-exposed $(3.33 \pm 0.21, \mathrm{p}=0.023)$ group when compared to the control $(2.16 \pm 0.16$, $\mathrm{p}=0.02)$ and vehicle $(2.00 \pm 0.36, \mathrm{p}=0.009)$ groups. The CUS+MYR-MEtreated group showed a significant decrease $(1.6 \pm 0.25, \mathrm{p}=0.004)$ in reference memory error when compared to CUS-exposed group.

Fig. $2 \mathrm{~b}$ shows the variation in the working memory error (reentry into the baited arm) among the groups ANOVA $(F(3,20)=5.68, p=0.006)$. The number of working memory error was increased $(2.5 \pm 0.22, \mathrm{p}=0.01)$ in CUS-exposed group when compared to control $(0.5 \pm 0.22, \mathrm{p}=0.019)$ and vehicle $(0.56 \pm 0.21, \mathrm{p}=0.03)$ groups, whereas CUS + MYR-ME--treated group showed pointedly decreased $(0.73 \pm 0.21, \mathrm{p}=0.006)$ working memory error when compared to CUS-exposed animals.

The time taken to visit all baited arms was prominently varied among the groups $(F(3,20)=338.103)$, and the results are shown in Fig. 2c. CUS group showed increased time $(153.16 \pm 2.77, \mathrm{p}=0.003)$ to visit all baited arm when compared to control $(56.83 \pm 2.32, \mathrm{p}=0.006)$ and vehicle (55 $\pm 2.47, \mathrm{p}=0.005)$ groups. However, MYR-ME-treated group showed significantly decreased time $(47.17 \pm 3.26, p=0.009)$ to visit all baited arms when compared to CUS-exposed group.

\section{Spontaneous alteration T-maze}

The difference in the number of days taken to attain $80 \%$ correct choice among the groups were determined by one-way ANOVA $(F(3,20)=45.38$, $\mathrm{p}=0.002$ ), the results have been summarized in Table 2 . Tukey's post hoc test revealed that the number of days taken to reach $80 \%$ correct response was more in CUS-exposed group $(4.3 \pm 0.21$ day, $\mathrm{p}=0.007)$ when compared to control $(1.66 \pm 0.21$ day, $\mathrm{p}=0.007)$ and vehicle $(1.83 \pm 0.16$ day, $\mathrm{p}=0.004)$ group. Whereas, the CUS+MYR-ME-treated group showed significantly fewer days taken $(1.83 \pm 0.16$ day, $\mathrm{p}=0.0031)$ when compared to CUS-exposed group.

Spontaneous alteration scoring is the percentage of correct choice, and this differed but not significant among groups $(F(3,20)=2.23)$. Spontaneous alteration scoring was less $(80.67 \pm 1.27 \%)$ in CUS- exposed animals when compared to control $(86.66 \pm 2.1 \%)$ and vehicle $(85.00 \pm 2.23 \%)$ groups. Whereas, CUS+MYR-ME-treated group showed increased (83.33 $\pm 2.10 \%)$ spontaneous alteration scoring.

There was a distinct variations in the average time taken for each entry among the groups $(F(3,20)=68.72, p=0.01)$. CUS-exposed animals showed increased time taken to visit each arm entry $(39.13 \pm 2.01 \mathrm{~s}$, $\mathrm{p}=0.006)$ when compared to control $(16.76 \pm 1.66 \mathrm{~s}, \mathrm{p}=0.001)$ and vehicle $(15.9 \pm 1.32, \mathrm{p}=0.003)$ groups, this was significantly decreased in CUS+MYR-ME-treated animals $(10.41 \pm 0.94 \mathrm{~s}, \mathrm{p}=0.001)$ when compared to CUS-exposed group.

Memory retention was varied among the groups $(F(3,20)=24.74$, $\mathrm{p}=0.008$ ). Memory retention scoring was significantly less in CUSexposed $(51.66 \pm 4.77 \%, \mathrm{p}=0.004)$ group when compared to control $(83.33 \pm 2.10 \%, \mathrm{p}=0.01)$ and vehicle $(81.66 \pm 1.66 \%, \mathrm{p}=0.01)$ groups, whereas CUS+MYR-ME-treated group showed significantly $(\mathrm{p}<0.05)$ increased memory retention scoring $(80.10 \pm 2.58 \%, \mathrm{p}=0.002)$ when compared to CUS-exposed group.

Average time taken for each entry during memory retention scoring significantly differed between the groups $(F(3,20)=169.02$, $\mathrm{p}=0.02$ ). CUS-exposed animals showed significantly more time taken $(56.09 \pm 2.82 \mathrm{~s}, \mathrm{p}=0.02)$ to visit each arm entry during memory retention scoring when compared to control $(15.75 \pm 0.85 \mathrm{~s}, \mathrm{p}=0.02)$ and vehicle $(15.17 \pm 1.36 \mathrm{~s}, \mathrm{p}=0.03)$ groups. MYR-ME-treated animals have taken significantly $(\mathrm{p}<0.05)$ less time took $(8.1 \pm 0.82 \mathrm{~s}, \mathrm{p}=0.01)$ when compared to CUS-exposed animals.

\section{Novel object recognition test}

There was a statistically significant difference in novel exploration between groups as determined by one-way ANOVA $(\mathrm{F}(3,20)=77.32$, $\mathrm{p}=0.03$ ), this is summarized in Fig. 3 . Tukey's post hoc test revealed that CUS-exposed animals showed significantly decreased $(26.62 \pm 1.64 \mathrm{~s}$, $\mathrm{p}=0.001)$ novelty preference when compared to control $(78.79 \pm 2.85 \mathrm{~s}$, $\mathrm{p}=0.007)$ and vehicle $(76.44 \pm 3.46 \mathrm{~s}, \mathrm{p}=0.006)$ groups. Whereas, the novelty preference was increased in CUS+MYR-ME-treated group $(70.86 \pm 2.70 \mathrm{~s}, \mathrm{p}=0.0043)$ when compared to CUS-exposed group.

ECG

Fig. 4a represents that the difference in the heart rate between the groups $(\mathrm{F}(3,20)=83.08, \mathrm{p}=0.02)$, the result shown in Tukey post hoc test revealed that CUS-exposed animals were showed significant increase in heart rate $(669.33 \pm 30.80 \mathrm{bpm}, \mathrm{p}=0.001)$ when compared to control $(395.66 \pm 25.65 \mathrm{bpm}, \mathrm{p}=0.005)$ and vehicle $(399.00 \pm 34.29 \mathrm{bpm}$, $\mathrm{p}=0.003$ ), the heart rate was significantly decreased in CUS+MYR-MEtreated animals $(426.66 \pm 30.80 \mathrm{bpm}, \mathrm{p}=0.001)$ when compared to CUSexposed animals.

R-R interval was markedly varied among the groups $(F(3,20)=9.06$, $\mathrm{p}=0.001$ ), this is shown in Fig. 4b. There was a significant decrease in CUS-exposed animals R-R interval rate $(0.19 \pm 0.03 \mathrm{~s}, \mathrm{p}=0.003)$ when compared to control $(0.42 \pm 0.03 \mathrm{~s}, \mathrm{p}=0.003)$ and vehicle $(0.45 \pm 0.05 \mathrm{~s}$, $\mathrm{p}=0.001)$. Whereas, the $\mathrm{R}-\mathrm{R}$ interval was significantly increased in CUS+MYR-ME-treated animals $(0.44 \pm 30.03 \mathrm{~s}, \mathrm{p}=0.002)$ when compared to CUS-exposed animals.

Table 2: Spontaneous alteration T-maze test

\begin{tabular}{lllll}
\hline Parameters & CON & VEH & CUS & CUS+MYR-ME \\
\hline Days taken to attain 80\% (days) & $1.66 \pm 0.21$ & $1.83 \pm 0.16^{\mathrm{c}}$ & $4.3 \pm 0.21^{\mathrm{a}, \mathrm{b}, \mathrm{d}}$ & $1.83 \pm 0.16^{\mathrm{c}}$ \\
Spontaneous alteration scoring (\%) & $86.66 \pm 2.10$ & $85.00 \pm 2.23$ & $80.67 \pm 1.27$ & $83.33 \pm 2.10$ \\
Average time taken to each entry (s) & $16.76 \pm 1.66$ & $15.9 \pm 1.32^{\mathrm{c}}$ & $39.13 \pm 2.01^{\mathrm{a}, \mathrm{b}, \mathrm{d}}$ & $10.41 \pm 0.94^{\mathrm{c}}$ \\
Memory retention scoring (\%) & $83.33 \pm 2.10$ & $81.66 \pm 1.66^{\mathrm{c}}$ & $51.66 \pm 4.77^{\mathrm{a}, \mathrm{b}, \mathrm{d}}$ & $80.10 \pm 2.58^{\mathrm{c}}$ \\
Average time taken for memory retention scoring (s) & $15.75 \pm 0.85$ & $15.17 \pm 1.36^{\mathrm{c}}$ & $56.09 \pm 2.82^{\mathrm{a}, \mathrm{b}, \mathrm{d}}$ & $8.1 \pm 0.82^{\mathrm{a}, \mathrm{b}, \mathrm{c}}$ \\
\hline
\end{tabular}

Describes parameters studied in the spontaneous alteration T-maze. The data were expressed as mean \pm SEM. "a" represents significance level when compared to control,

"b" represents compared to vehicle, "c" compared to unpredictable stress group, and " $\mathrm{d}$ " represents compared to stress with MYR-ME treated and the value $p<0.05$ is considered as statistically significant. SEM: Standard error of mean, CUS: Chronic unpredictable stress, MYR: Myricetin, ME: Microemulsion 


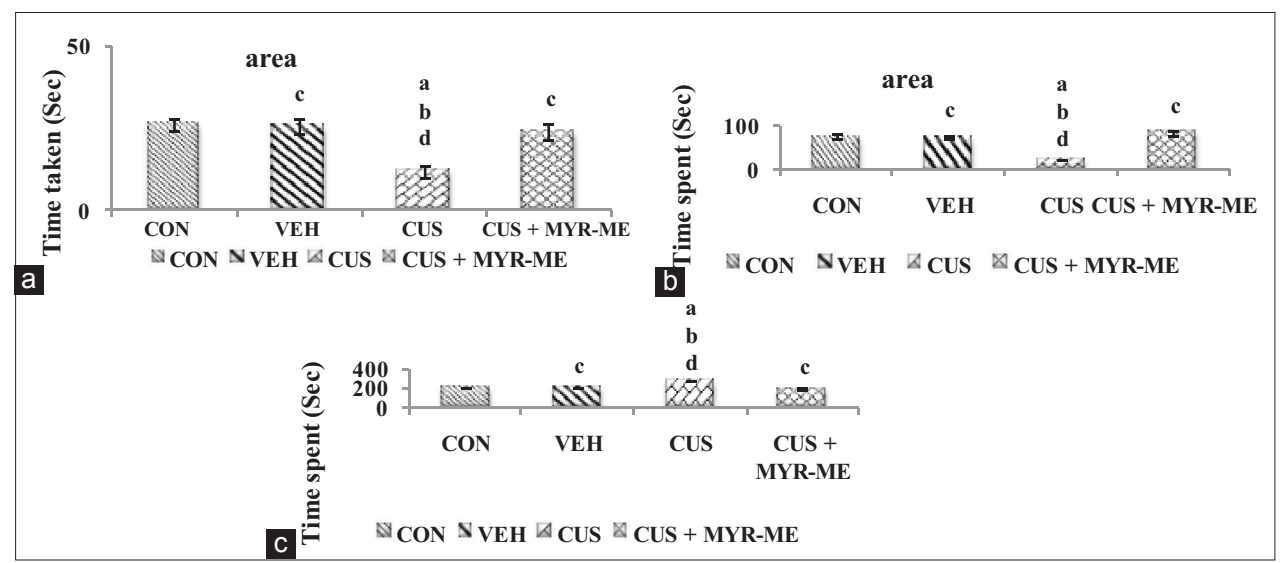

Fig. 1: Light and dark box test, initial latency to enter into a dark area (a), time spent in bright areas (b) and time spent in a dark area (c). The data were expressed as mean \pm standard error of the mean. "a" represents significance level when compared to control, "b" represents compared to vehicle, "c" represents compared to unpredictable stress group, and "d" represents compared to stress with myricetinmicroemulsion treated and the value $\mathbf{p}<0.05$ is considered as statistically significant

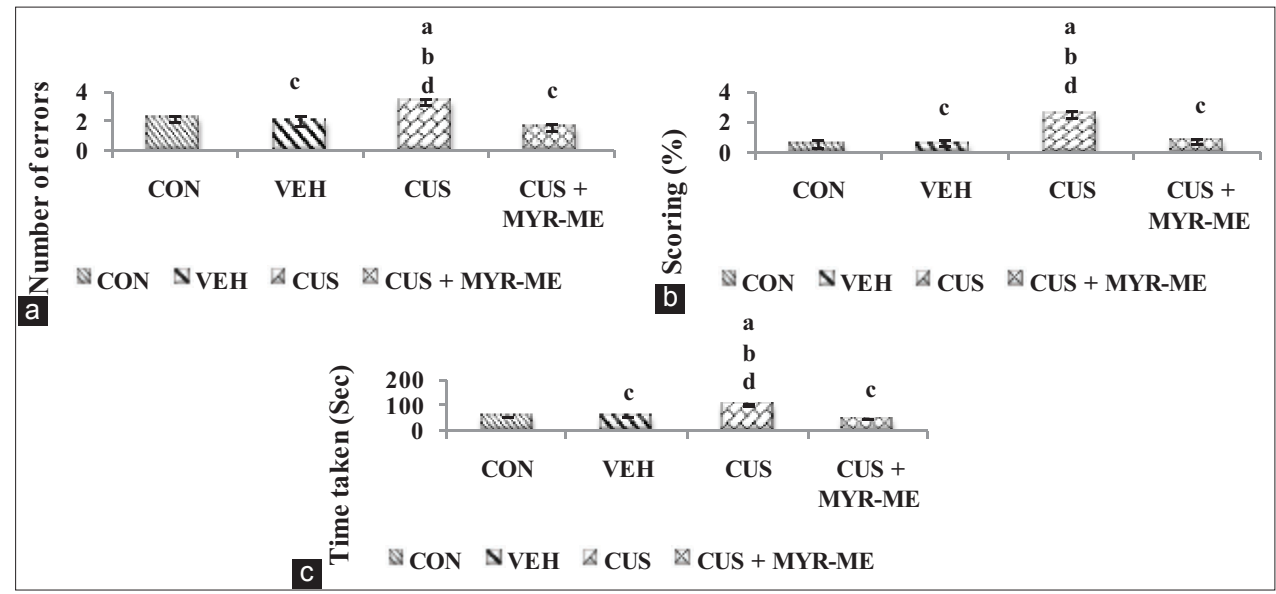

Fig. 2: Eight-arm radial maze, working memory error (a), reference memory (b) and time was taken (c). The data were expressed as mean \pm standard error of the mean. "a" represents significance level when compared to control, "b" represents compared to vehicle, "c" represents compared to unpredictable stress group, and " $\mathrm{d}$ " represents compared to stress with myricetin-microemulsion treated and the value $\mathbf{p}<0.05$ is considered as statistically significant

The RMSSD was diverged among groups $(F(3,20)=31.58, p=0.02)$, the result were showed in Fig. 4c. RMSSD was significantly decreased (3.31 $\pm 0.24 \mathrm{~ms}, \mathrm{p}=0.009$ ) in CUS-exposed animals when compared to control $(7.9 \pm 0.42 \mathrm{~ms}, \mathrm{p}=0.005)$ and vehicle $(7.66 \pm 0.44 \mathrm{~ms}, \mathrm{p}=0.006)$. Whereas, CUS+MYR-ME-treated group showed significant increase $(6.83 \pm 0.37 \mathrm{~ms}, \mathrm{p}=0.005)$ in RMSSD value when compared to CUSexposed animals.

\section{EEG}

There was a statistically significant difference between the groups in the brain electrical activity; the results were summarized in Table 3. The CUS-exposed animals were showed significant increase in frontal and occipital lobe frequency and amplitude when compared to control frequency, amplitude, and vehicle frequency. However, CUS+MYR-MEtreated animals showed significantly decreased frontal and occipital lobe frequency and amplitude when compared to CUS-exposed animals.

\section{Plasma corticosterone}

Plasma corticosterone levels have significantly varied among the groups; the data are summarized in Fig. 5. The significant increase level of corticosterone $(85.83 \pm 7.57 \mathrm{ng} / \mathrm{ml}, \mathrm{p}=0.003)$ was observed in the CUS-exposed animals when compared to control $(40.16 \pm 4.15 \mathrm{ng} / \mathrm{ml}, \mathrm{p}=0.007)$ and vehicle $(39.33 \pm 5.33 \mathrm{ng} / \mathrm{ml}, \mathrm{p}=0.004)$.
Whereas, CUS+MYR-ME-treated animals showed significantly decreased $(44.5 \pm 7.87 \mathrm{ng} / \mathrm{ml}, \mathrm{p}=0.001)$ corticosterone level when compared to CUS-exposed animals.

\section{DISCUSSION}

Stress has become an integral part of human life, and stressful experience disturbs the body's physiological function by elevating the secretions of stress hormones. TThe present study demonstrates that, increased level of corticosterone in the CUS-exposed animals may deteriorate the brain damage and subsequently which may leads to cognitive dysfunction, learning and memory impairment and also alters the electrophysiological activities. The results from the present study confirm our hypothesis that MYR-ME may actively prevent or protect cognitive dysfunction and anxiety-like behavior and may be restored brain electrical activities and HR induced by CUS. This is evident by decreased corticosterone levels, anxiety-like behavior, HR, brain waves amplitude, frequency and improved decision-making ability, and novel exploration, and learning and memory were observed in CUS with MYR-ME-treated group. Earlier studies reported that polyphenols has a great attention in the recent days, and it has been used to treat for many diseases and disorders including cognitive dysfunction [30]. MYR is one of the key constituents present in the various human foods and has numerous health beneficial activities such as anticancer, antioxidant, 
Table 3: Electroencephalogram

\begin{tabular}{lllll}
\hline Parameters & CON & VEH & CUS & CUS+MYR-ME \\
\hline Frontal lobe frequency $(H z)$ & $15.06 \pm 1.61$ & $13.24 \pm 0.25^{\mathrm{c}}$ & $26.21 \pm 0.365^{\mathrm{a}, \mathrm{b}, \mathrm{d}}$ & $15.24 \pm 1.04^{\mathrm{c}}$ \\
Frontal lobe amplitude $(\mu \mathrm{v})$ & $17.91 \pm 1.61$ & $17.31 \pm 1.27^{\mathrm{c}}$ & $44.72 \pm 3.47^{\mathrm{a}, \mathrm{b}, \mathrm{d}}$ & $16.16 \pm 0.48^{\mathrm{c}}$ \\
Occipital lobe frequency $(\mathrm{Hz})$ & $17.22 \pm 0.99$ & $15.65 \pm 0.67^{\mathrm{c}}$ & $26.96 \pm 0.74^{\mathrm{a}, \mathrm{b}, \mathrm{d}}$ & $16.38 \pm 1.04^{\mathrm{c}}$ \\
Occipital lobe amplitude $(\mu \mathrm{v})$ & $19.32 \pm 1.61$ & $21.04 \pm 3.52^{\mathrm{c}}$ & $39.58 \pm 4.22^{\mathrm{a}, \mathrm{b}, \mathrm{d}}$ & $20.94 \pm 1.65^{\mathrm{c}}$ \\
\hline
\end{tabular}

Describes parameters studied in the electroencephalogram. The data were expressed as mean \pm SEM. "a" represents significance level when compared to control, "b" represents compared to vehicle, "c" compared to unpredictable stress group, and " $\mathrm{d}$ " represents compared to stress with MYR-ME-treated and the value $\mathrm{p}<0.05$ is considered as statistically significant. SEM: Standard error of mean, CUS: Chronic unpredictable stress, MYR: Myricetin, ME: Microemulsion

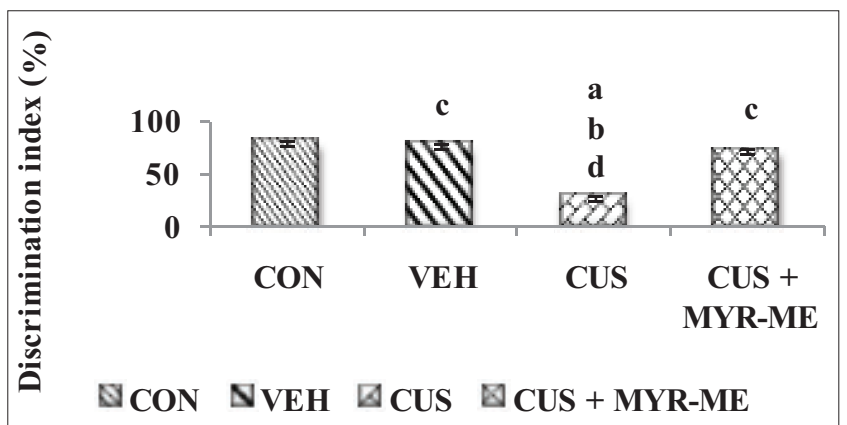

Fig. 3: Novel object recognition test. The data were expressed as mean \pm standard error of the mean. "a" represents significance level when compared to control, "b" represents compared to vehicle, "c" represents compared to unpredictable stress group, and " $d$ " represents compared to stress with myricetinmicroemulsion treated and the value $p<0.05$ is considered as statistically significant

antihypertensive, and immunomodulatory [16] though, due its partial polar solubility nature, it is inaccessible to across the BBB, this limits its use to CNS-related problems. Hence, an alternative delivery system used in the present study.

The CUS-exposed animals showed higher anxiety-like behavior in the open-field and light/dark test, this may be due to alterations in HPA axis hormone and induce elevation of plasma corticosterone, this was restored in CUS+MYR-ME-treated animals which may be the modulatory effect of MYR-ME by reducing plasma corticosterone levels. This is supported by previous findings, chronic restraint and CUS showed anxiety-like behavior, and this was reduced in the MYR-administrated group [1,31,32]. Riaz et al. (2015) reported that CUS-exposed animals showed impaired spatial memory in the eight-arm radial maze [33], this is consistent with the present findings. Spontaneous alteration T-maze used to assess the cognitive ability such as decision-making and memory retention ability of the animals. Impaired decision-making, memory retention ability, and spatial memory were observed in CUSexposed animals, this was improved in MYR-ME-treated animals, and this would be due to decreased level of corticosterone which may inhibit the glutamate-induced excitotoxicity which may favor learning and memory and cognitive function by increasing the neuronal plasticity and neurogenesis. An earlier study reported that stress alters the synaptic plasticity and learning memory by altering glutamate transmission [34]. Further, it is confirmed that MYR has capable of inhibiting the glutamate release in the cortical nerve terminal by suppressing of presynaptic $\mathrm{Ca}^{2+}$ influx [35]. Recent findings reported that MYR has capable of ameliorates rotenone-induced parkinsonism in drosophila [36]. Malathi et al. also reported that animals which were exposed to chronic restraint stress showed impaired learning and memory which was restored by Michelia champaca extract [37]. Novel object recognition test is used to evaluate the recognition memory/novel exploratory behavior. The animal's capacity to recognize and recollect the objects is tested by exposing them to two identical objects in an open field and then later exposing them again to the field with one of the familiar objects from the first trial and other one novel object and subsequently comparing the time spent each in each objects [38]. Recognition memory is the component of hippocampal function, in the present study, CUS-exposed animals showed impaired recognition memory, this would be over activation of HPA axis, hormone might have affected the hippocampus, and this was further confirmed by Vidyashree et al. [37]. Although the high base line levels of corticosterone in the CUS-exposed animals may subsequently increase when exposed to the novel object, this may further worsen recognition memory. The similar results were observed in the earlier studies, unpredictable subchronic stress affects memory through increased glucocorticoid levels which might have implications for brain plasticity and behavioral changes following the stress in rat's hippocampus [39]. Recognition memory was improved in MYR-ME treated, the possible mechanism could be decreasing corticosterone levels and increasing brain-derived neurotropic factor (BDNF) levels, previous study also reported that chronic restraint stress increased corticosterone levels and decreased BDNF levels, and this was normalized in the MYR treatment animals by attributing to MYRmediated antioxidative stress in the hippocampus [32].

Electrophysiological studies (ECG and EEG) have been used as a biomarker for ANS and CNS activity associated with mental stress [40]. $\mathrm{HRV}$ analysis is an established tool to estimate cardiac autonomic regulation in humans and animal models. Traditional HRV methods fall under the broader description of being either "time-domain" or "frequency domain" analyses. Among time domain, measures of HRV are HR, R-R intervals, and RMSSD which estimates the parasympathetic activity [10]. Park et al. reported that unpredictable mild stress showed significantly decreased time domain analysis such as R-R interval and RMSSD in HRV index, this is consistent with our present findings, CUSexposed animals showed significantly increased HR and significantly decreased R-R intervals and RMSSD were observed, and this shows that there would be dysfunction in the ANS [40]. The possible reason would be the elevated corticosterone levels, which may alter the HR and HRV index; this was normalized in the MYR-ME-treated animals. An EEG is the test to measure the brain electrical activities, earlier study reported that the brain waves pattern based on the frequency, delta $(1-5 \mathrm{~Hz})$, theta (6-8 Hz), alpha $1(9-10 \mathrm{~Hz})$, alpha $2(11-12 \mathrm{~Hz})$, beta $1(13-$ $17 \mathrm{~Hz})$, beta $2(18-20 \mathrm{~Hz})$, beta $3(21-30 \mathrm{~Hz})$, and beta $4(31-100 \mathrm{~Hz})$, alpha, and beta 1 are the mostly predominantly present in the active rats [41]. The increased level of corticosterone stimulates the release of glutamate which may augment the frequency by altering excitatory postsynaptic potential in the hippocampal pyramidal neurons [42]. Further, increased cerebral blood flow during chronic restraint stress impaired the BBB and caused the changes in the EEG waves [43]. These would be the possible reason for significantly increased frontal lobe frequency (beta 3), amplitude and occipital lobe frequency (beta 3), and amplitude in the CUS-exposed animals. However, MYR-ME treated animals restored the electrophysiological alterations caused by CUS exposure. The possible mechanism of attenuating of stress in MYR-MEtreated animals would be increased bioavailability of drug delivery, the potential decreasing effect of corticosterone, and glutamate followed by the inhibition cell excitotoxicity [44]

The characteristic feature of the study is that, ME formulation is one of the suitable alternative drug delivery methods for polar soluble or partially polar soluble compounds to enhance the drug delivery to the brain, and we have performed spontaneous alteration T-maze test 


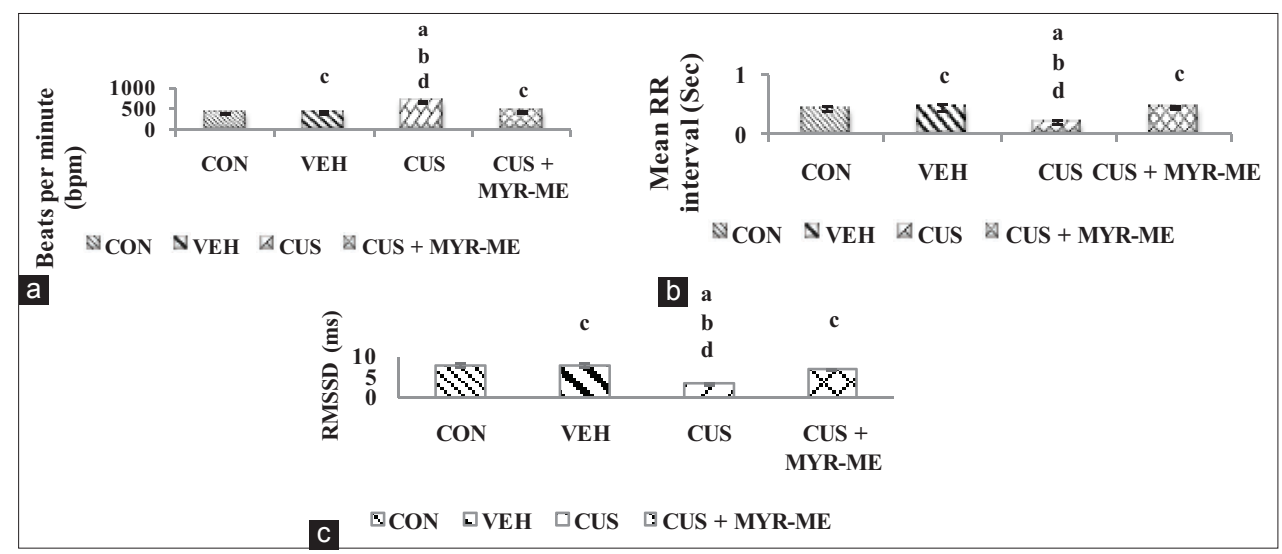

Fig. 4: Describes electrocardiography (a) represents heart rate, (b) RR intervals, and (c) RMSSD. The data were expressed as mean \pm standard error of the mean. "a" represents significance level when compared to control, " $b$ " represents compared to vehicle, "c" represents compared to unpredictable stress group, and " $\mathrm{d}$ " represents compared to stress with myricetin-microemulsion treated and the value $\mathbf{p}<0.05$ is considered as statistically significant

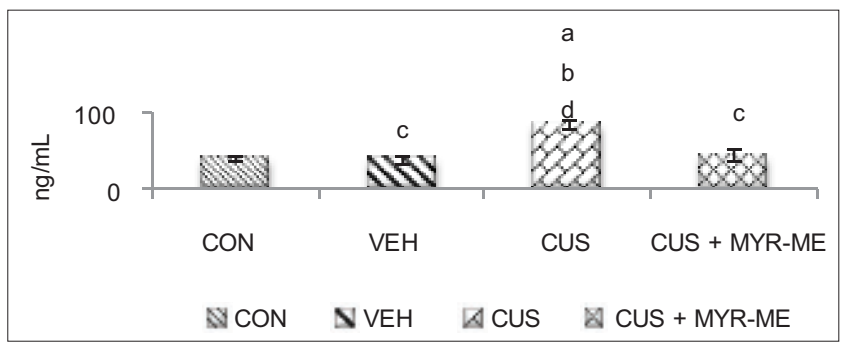

Fig. 5: Plasma corticosterone levels. The data were expressed as mean \pm standard error of the mean. "a" represents significance level when compared to control, "b" represents compared to vehicle, "c" represents compared to unpredictable stress group, and " $d$ " represents compared to stress with myricetinmicroemulsion treated and the value $\mathbf{p}<0.05$ is considered as statistically significant

to assess cognitive functions such as decision-making and memory retention ability in the CUS-exposed rats, and also electrophysiological techniques (ECG and EEG) are the potential biomarkers to assess the mental stress. However, the study also has limitations; we did not measure the catecholamines and other neurotransmitters and also molecular mechanism behind the ameliorative effect MYR-ME. Hence, it is necessary to carry out neurotransmitter estimation and genomic and proteomic expressions to identify the clear mechanism behind the ameliorative effect of MYR-ME, this will be carried out in future research.

\section{CONCLUSION}

The present study suggests that CUS has a detrimental effect on cognitive function, anxiety behavior, brain electrical activity, and HR. Although MYR has biologically diverse action, due to its partial polar soluble nature, it is necessary to find a better alternative delivery method to target the brain. Hence, the ME delivery system was developed and used in the present study. The ME form of MYR was potentially ameliorated the cognitive function, anxiety-related behavior, and electrophysiological activities induced by CUS. The ME form MYR efficiently act as anxiolytic and nootropic actions.

\section{ACKNOWLEDGMENT}

The financial assistance from the University of Madras to carry out this research work and the financial support provided in the form senior research fellowship (SRF) by the Indian Council of Medical Research are greatly acknowledged.

\section{AUTHOR CONTRIBUTIONS}

Conceptualization: SG RR Data curation: SG RR

Formal analysis: SG RR DKM.

Investigation: SG RR DKM

Methodology: SG RR DKM PP KCS.

Project administration: SG RR PP KCS.

Software: SG RR KCS PP.

Supervision: SG RR DKM PP.

Validation: SG RR

Visualization: SG RR DKM PP KCS

Writing - original draft: SG DKM.

Writing - review and editing: SG RR DKM KCS.

\section{CONFLICTS OF INTEREST}

The author has no conflict of interest.

\section{REFERENCES}

1. Gouirand AM, Matuszewich L. The effects of chronic unpredictable stress on male rats in the water maze. Physiol Behav 2005;86:21-31.

2. Gopalan G, Bannon W, Dean-Assael K, Fuss A, Gardner L, LaBarbera B, et al. Multiple family groups: An engaging intervention for child welfare-involved families. Child Welfare 2011;90:135-56.

3. Vyas A, Mitra R, Shankaranarayana Rao BS, Chattarji S. Chronic stress induces contrasting patterns of dendritic remodeling in hippocampal and amygdaloid neurons. J Neurosci 2002;22:6810-8.

4. Rao VR, Finkbeiner S. NMDA and AMPA receptors: Old channels, new tricks. Trends Neurosci 2007;30:284-91.

5. Wang $\mathrm{H}, \mathrm{Hu} \mathrm{Y}$, Tsien JZ. Molecular and systems mechanisms of memory consolidation and storage. Prog Neurobiol 2006;79:123-35.

6. Park CR, Campbell AM, Diamond DM. Chronic psychosocial stress impairs learning and memory and increases sensitivity to yohimbine in adult rats. Biol Psychiatry 2001;50:994-1004

7. Mizoguchi K, Yuzurihara M, Ishige A, Sasaki H, Chui DH, Tabira T, et al. Chronic stress induces impairment of spatial working memory because of prefrontal dopaminergic dysfunction. J Neurosci 2000;20:1568-74.

8. Liu D, Zhang Q, Gu J, Wang X, Xie K, Xian X, et al. Resveratrol prevents impaired cognition induced by chronic unpredictable mild stress in rats. Prog Neuropsychopharmacol Biol Psychiatry 2014;49:21-9.

9. Matuszewich L, Karney JJ, Carter SR, Janasik SP, O’Brien JL, Friedman RD, et al. The delayed effects of chronic unpredictable stress on anxiety measures. Physiol Behav 2007;90:674-81.

10. Zhong X, Hilton HJ, Gates GJ, Jelic S, Stern Y, Bartels MN, et al. Increased sympathetic and decreased parasympathetic cardiovascular modulation in normal humans with acute sleep deprivation. J Appl Physiol 2005;98:2024-32.

11. Loganathan S, Rathinasamy S. Alteration in memory and electroencephalogram waves with sub-acute noise stress in albino rats 
and safeguarded by scoparia dulcis. Pharmacogn Mag 2016;12:S7-S13 .

12. Kesselheim AS, Hwang TJ, Franklin JM. Two decades of new drug development for central nervous system disorders. Nat Rev Drug Discov 2015;14:815-6.

13. Ong KC, Khoo HE. Biological effects of myricetin. Gen Pharmacol 1997;29:121-6.

14. Yoshikawa M, Shimada H, Nishida N, Li Y, Toguchida I, Yamahara J, et al. Antidiabetic principles of natural medicines. II. Aldose reductase and alpha-glucosidase inhibitors from brazilian natural medicine, the leaves of Myrcia multiflora DC. (Myrtaceae): Structures of myrciacitrins I and II and myrciaphenones A and B. Chem Pharm Bull (Tokyo) 1998:46:113-9.

15. Chen W, Li Y, Li J, Han Q, Ye L, Li A. Myricetin affords protection against peroxynitrite-mediated dna damage and hydroxyl radical formation. Food Chem Toxicol 2011;49:2439-44.

16. Semwal DK, Semwal RB, Combrinck S, Viljoen A. Myricetin: A dietary molecule with diverse biological activities. Nutrients 2016;8:90.

17. Hagenacker T, Hillebrand I, Wissmann A, Büsselberg D, Schäfers M. Anti-allodynic effect of the flavonoid myricetin in a rat model of neuropathic pain: Involvement of p38 and protein kinase C mediated modulation of $\mathrm{ca}^{2}+$ channels. Eur J Pain 2010;14:992-8

18. Mohan M, Jadhav SS, Kasture VS, Kasture SB. Effect of myricetin on behavioral paradigms of anxiety. Pharm Biol 2009:47:927-31.

19. Yao Y, Lin G, Xie Y, Ma P, Li G, Meng Q, et al. Preformulation studies of myricetin: A natural antioxidant flavonoid. Pharmazie 2014;69:19-26.

20. Hoar TP, Schulman JH. Transparent water-in-oil dispersions: The oleopathic hydro-micelle. Nature 1943;152:102-3.

21. Wang S, Ye T, Zhang X, Yang R, Yi X. Myricetin microemulsion for oral drug delivery: Formulation optimization, in situ intestinal absorption and in-vivo evaluation. Asian J Pharm Sci 2013;8:18-25.

22. Katz RJ. Animal model of depression: Pharmacological sensitivity of a hedonic deficit. Pharmacol Biochem Behav 1982;16:965-8.

23. Prut L, Belzung C. The open field as a paradigm to measure the effects of drugs on anxiety-like behaviors: A review. Eur J Pharmacol 2003;463:3-3.

24. Arrant AE, Schramm-Sapyta NL, Kuhn CM. Use of the light/dark test for anxiety in adult and adolescent male rats. Behav Brain Res 2013;256:119-27.

25. Olton DS, Samuelson RJ. Remembrance of places passed: Spatial memory in rats. J Exp Psychol Anim Behav Process 1976;2:97-116.

26. Deacon RM, Rawlins JN. T-maze alternation in the rodent. Nat Protoc 2006; $1: 7-12$

27. Antunes M, Biala G. The novel object recognition memory: Neurobiology, test procedure, and its modifications. Cogn Process 2012;13:93-110.

28. Thandapilly SJ, Louis XL, Behbahani J, Movahed A, Yu L, Fandrich $\mathrm{R}$, et al. Reduced hemodynamic load aids low-dose resveratrol in reversing cardiovascular defects in hypertensive rats. Hypertens Res 2013;36:866-72.

29. Choudhary AK, Sundareswaran L, Devi RS. Effects of aspartame on the evaluation of electrophysiological responses in wistar albino rats. J Taibah Univ Sci 2016;10:505-12.

30. Rai AR, Madhyastha S, Prabhu LV, Saralaya VV, Sahu SS. Resveratrol reverses the restraint stress-induced cognitive dysfunction involving brain antioxidant system in rats. Int J Pharm Pharm Sci 2014;6:768-72.

31. Chiba S, Numakawa T, Ninomiya M, Richards MC, Wakabayashi C, Kunugi H. Chronic restraint stress causes anxiety-and depression-like behaviors, downregulates glucocorticoid receptor expression, and attenuates glutamate release induced by brain-derived neurotrophic factor in the prefrontal cortex. Prog Neuropsychopharmacol Biol Psychiatry. 2012;39:112-9.

32. Ma Z, Wang G, Cui L, Wang Q. Myricetin attenuates depressant-like behavior in mice subjected to repeated restraint stress. Int J Mol Sci 2015; $16: 28377-85$

33. Riaz MS, Bohlen MO, Gunter BW, Quentin H, Stockmeier CA, Paul IA, et al. Attenuation of social interaction-associated ultrasonic vocalizations and spatial working memory performance in rats exposed to chronic unpredictable stress. Physiol Behav 2015;152:128-34.

34. Popoli M, Yan Z, McEwen BS, Sanacora G. The stressed synapse: The impact of stress and glucocorticoids on glutamate transmission. Nat Rev Neurosci 2011;13:22-37.

35. Chang Y, Chang CY, Wang SJ, Huang SK. Myricetin inhibits the release of glutamate in rat cerebrocortical nerve terminals. J Med Food 2015;18:516-23.

36. Dhanraj V, Manivasagam T, Karuppaiah J. Myricetin isolated from turbinaria ornata ameliorates rotenone induced parkinsonism in drosophila melanogaster. Int J Pharm Pharm Sci 2017;9:1-6.

37. Vidyashree HM, Malathi S, Ravindran R. Impaired object recognition memory and acetylcholinesterase activity in animal model of posttraumatic stress disorder-restored by Eclipta alba Linn. A dietary herb. Asian J Pharm Clin Res. 2016;9: 117-121.

38. Singh P, Thakur MK. Reduced recognition memory is correlated with decrease in DNA methyltransferase 1 and increase in histone deacetylase 2 protein expression in old male mice. Biogerontology 2014;15:339-46.

39. Kazlauckas V, Kalinine E, Leke R, Oses JP, Nunes F, Espinosa J, et al. Distinctive effects of unpredictable subchronic stress on memory, serum corticosterone and hippocampal BDNF levels in high and low exploratory mice. Behav Brain Res 2011;218:80-6.

40. Hollenstein T, McNeely A, Eastabrook J, Mackey A, Flynn J. Sympathetic and parasympathetic responses to social stress across adolescence. Dev Psychobiol 2012;54:207-14.

41. van Lier H, Drinkenburg WH, van Eeten YJ, Coenen AM. Effects of diazepam and zolpidem on EEG beta frequencies are behavior-specific in rats. Neuropharmacology 2004;47:163-74.

42. Karst H. Corticosterone slowly enhances miniature excitatory postsynaptic current amplitude in mice CA1 hippocampal cells. J Neurophysiol 2005;94:3479-86

43. Sharma HS, Dey PK. EEG changes following increased blood-brain barrier permeability under long-term immobilization stress in young rats. Neurosci Res 1988;5:224-39.

44. Shimmyo Y, Kihara T, Akaike A, Niidome T, Sugimoto H. Three distinct neuroprotective functions of myricetin against glutamate-induced neuronal cell death: Involvement of direct inhibition of caspase-3. J Neurosci Res 2008;86:1836-45. 